\title{
A novel detector based on EM algorithm for multiple-mode OFDM with index modulation
}

\author{
Hua Qing ${ }^{1,2}$, Hua $\mathrm{Yu}^{1,2^{*}} \mathbb{D}$, Miaowen Wen ${ }^{1}$, Fangjiong Chen ${ }^{1}$ and Fei $\mathrm{Ji}^{1}$
}

\begin{abstract}
Multiple-mode orthogonal frequency division multiplexing with index modulation (MM-OFDM-IM) is a promising multiple-carrier modulation technique, which activates all OFDM subcarriers to transmit multiple distinguishable modes (or signal constellations, alternatively) and carries additional information with the full permutation of the modes, enjoying superior error performance and high spectral efficiency. In this paper, we propose a novel detector tailored to MM-OFDM-IM based on the expectation maximization (EM) algorithm to further improve the detection performance. The idea is to first select the signal constellation points candidates for any given mode permutation by applying the EM algorithm and then determine the most-likely mode permutation as well as the associated signal constellation points by minimizing the Euclidean distance. The effectiveness of the EM detector has been verified in terms of bit error rate (BER) performance by Monte Carlo simulations.
\end{abstract}

Keywords: BER, Expectation maximization (EM), Index modulation, Maximum-likelihood (ML), OFDM

\section{Introduction}

Recently, index modulation (IM) technique, which is an effective digital modulation strategy that carries information bits through the index of some building block of a communication system, emerges. Due to its high spectral-and energy-efficiency, IM has been widely considered for future wireless networks [1-4]. More recently, the concept of IM has been transplanted to orthogonal frequency division multiplexing (OFDM) subcarriers, giving birth to OFDM-IM [5, 6]. Typically, OFDM-IM divides the incoming information bits into two parts, of which one chooses a subset of subcarriers to be active leaving the others idle, and the other is modulated by conventional phase shift keying (PSK) or quadrature amplitude modulation. It has been verified that OFDMIM provides better achievable rate and BER performance than the classical OFDM $[7,8]$.

*Correspondence: yuhua@scut.edu.cn

${ }^{1}$ The National Engineering Technology Research Center for Mobile Ultrasonic Detection, South China University of Technology, 510641 Guangzhou, China ${ }^{2}$ Key Laboratory of Marine Environmental Survey Technology and Application, Ministry of Natural Resources, 510300 Guangzhou, China
Inspired by OFDM-IM, researchers have denoted more and more efforts in developing multiple-carrier IM techniques. In [9], the authors have applied the idea of IM concept to both the in-phase and quadrature (IQ) dimensions of the OFDM signal, proposing OFDM-IM-IQ, which can further improve the spectral efficiency. In [10], the multiple-input multiple-output OFDM-IM (MIMOOFDM-IM), which integrates the MIMO paradigm with OFDM-IM, has been proposed, providing flexibility in the tradeoff between spectral efficiency and bit error rate (BER) performance. The dual-mode OFDM-IM (DMOFDM-IM) has been proposed in [11], unlike OFDM-IM, all subcarriers in DM-OFDM-IM are active and divided into two parts that are modulated with two distinguishable signal constellations. In [12], DM-OFDM-IM has been generalized into multiple-mode OFDM-IM (MM-OFDMIM), where all subcarriers are activated as DM-OFDM-IM of which each is assigned a different mode (or say signal constellation), and the IM bits are conveyed by the full permutation of all modes. It is reported therein that MMOFDM-IM outperforms all existing multiple-carrier IM schemes in terms of both spectral efficiency and BER. 
This paper focuses on the design of an effective detector for the MM-OFDM-IM system. So far, there have been different kinds of detectors designed for OFDM-based IM schemes under the perfect channel. In [5], the lowcomplexity log-likelihood ratio (LLR) detector and the optimal maximum-likelihood (ML) detector for OFDMIM have been proposed, where the former achieves nearML performance with much lower complexity than the latter. An ML detector for OFDM-IM-IQ with low complexity has been proposed in [13], which enables IQindependent subcarrier-wise (SW) detection. In [14], a detector for MIMO-OFDM-IM based on the sequential Monte Carlo theory has been designed with lower complexity. An LLR detector with reduced complexity for DM-OFDM-IM has been proposed in [11], which demodulates the signals in an iterative manner. In [12], an SW detector and a low-complexity ML (LCML) detector for MM-OFDM-IM with even lower complexity have been proposed, where the latter is implemented with a Viterbilike algorithm and the former is designed based on the idea of LLR detection that is found equivalent to the minimum Euclidean distance detection.

The ML detection is a common method to estimate the system parameters based on the exact sampling data [15, 16]. However, in practice, some useful information carried by the sampling data is usually well-hidden, namely the data that is closely related to the results but not observed intuitively, this causes in the likelihood function cannot be manipulated directly, which means ML detection cannot be explored [17]. The EM algorithm can effectively solve this type of problem with unobserved data by constructing a local lower-bound of the likelihood function with the expectation step (E step) and maximize it with the maximum step ( $M$ step) in an iterative manner, and as a result, it has been widely applied to the field of wireless communications [18-20]. Motivated by this and to further explore the BER performance of the MM-OFDM-IM system with unknown channel since the detectors currently designed for MM-OFDM-IM system are under perfect channel, in this paper, we propose an EM detector to estimate the parameters of MM-OFDMIM signals under the unobserved data, namely channel impulse response (CIR), in two stages. The first stage is to locate the possible signal constellation points iteratively according to the EM algorithm for a given mode permutation. Then, all located signal constellation points for all mode permutations trials are included in a candidate set as the input of the second stage, which acts as a filter and outputs the most-likely correct signal constellation points with the minimum Euclidean distance to the received signal. Monte Carlo computer simulations are conducted, whose results show that the BER performance of the proposed EM detector is better than ML, SW, and LCML detectors, and obtains an up to $1.2 \mathrm{~dB}$ signal-to- noise ratio (SNR) gain over the $\mathrm{ML}$ detector in the high SNR region. To evaluate the efficiency of the proposed EM algorithm, we also measured the average number of iterations required for the EM detector to converge. The results indicate that the average number of iterations for the proposed EM detector convergence is very small, which is less than 2, and it decreases with the increase of the SNR.

The remainder of this paper is organized as follows. In Section 2, we first describe the system model of MMOFDM-IM, then introduce the principle of the proposed EM detector and analyze the computational complexity. In Section 3, the simulation results are demonstrated. Finally, the paper is concluded in Section 4.

Notation: Bold lowercase letters and bold uppercase letters indicate vectors and matrices, respectively. $(\cdot)^{H},(\cdot)^{T}$, and $(\cdot)^{-1}$ represent the Hermitian transpose, transpose, and inversion, respectively. $E\{\cdot\}$ represents the mathematical expectations. $\operatorname{Pr}(\cdot)$ indicates the probability of an event, and $f(\cdot)$ denotes the probability density function. $\operatorname{diag}(\cdot)$ represents the diagonalization. $\|\cdot\|$ stands for the Frobenius norm. $\lfloor\cdot\rfloor$ denotes the floor operation. $\mathbf{I}_{n}$ indicates the $n \times n$ identity matrix.

\section{Methods}

In this section, we first review the MM-OFDM-IM systems, and then design a detector based on the EM algorithm to estimate the system parameters of MM-OFDM-IM under the unobserved data CIR.

\subsection{System model of MM-OFDM-IM}

In MM-OFDM-IM systems, all the subcarriers are activated and each subcarrier will be transmitted with a specific mode; meanwhile, the full permutation of these modes will also be used to carry additional information bits. Figure 1 depicts the MM-OFDM-IM transmitter structure.

In Fig. 1, every $m$ bits form a subblock containing $n$ subcarriers, the $m$ bits are further divided into two parts, where $m_{1}$ bits constitute the first part and are used to determine the permutation of $n$ distinguishable modes $\left\{\mathcal{M}_{1}, \ldots, \mathcal{M}_{n}\right\}$, where $\mathcal{M}_{\gamma}$ is an $M$-ary constellations with $\gamma \in\{1, \ldots, n\}$, and we normalized the average power of $\left\{\mathcal{M}_{1}, \ldots, \mathcal{M}_{n}\right\}$ to unity. The method of mode selection for $M$-ary constellations has been also described in [12], which indicates that the optimal signal constellations for $n$ modes can be achieved by rotating the original $M$-ary constellation with angles $2 \pi(\gamma-1) / n M$. Assume that the permutation is $J=\left\{j_{1}, \ldots, j_{n}\right\}$, which implies the $n$ subcarriers in one subblock will employ $\left\{\mathcal{M}_{j_{1}}, \ldots, \mathcal{M}_{j_{n}}\right\}$ in order, where $j_{\gamma} \in\{1, \ldots, n\}$ refers to the index of the $\gamma$ th subcarrier. The parameter $m_{1}$ is set as $m_{1}=\left\lfloor\log _{2}(n !)\right\rfloor$ since $n$ ! is the number of all possible full mode permutations. The mapping between the $m_{1}$ bits and the permutation 


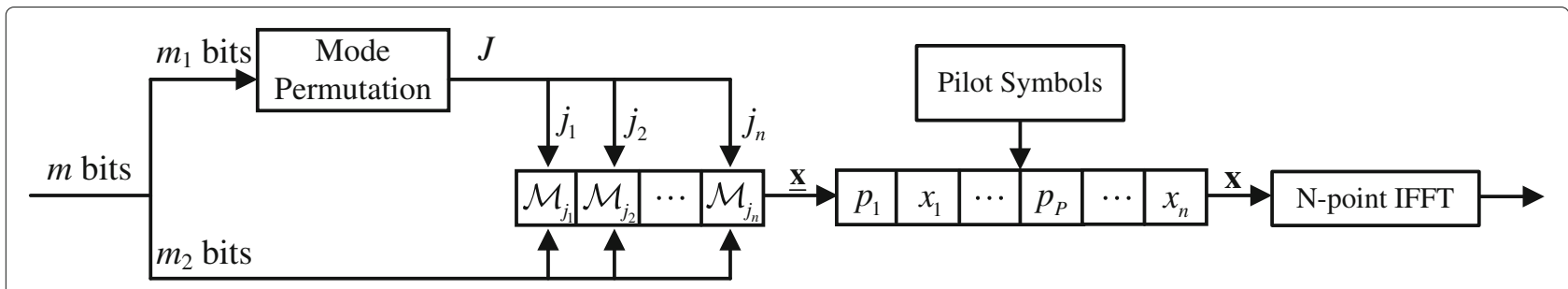

Fig. 1 The transmitter schematic diagram for MM-OFDM-IM systems

indices $\left\{j_{1}, \ldots, j_{n}\right\}$ can be performed through the permutation method or a look-up table [12]. The second part, comprised of $m_{2}=n \log _{2}(M)$ bits, determines $n$ information symbols $\underline{\mathbf{x}}=\left[x_{1}, \ldots, x_{n}\right]^{T}$ based on the selected mode permutation $J$, where $x_{\gamma} \in \mathcal{M}_{j_{\gamma}}$. Next, insert the pilot symbols $\mathbf{p}=\left\{p_{1}, p_{2}, \ldots, p_{P}\right\}$ at equal intervals, note that since we insert the pilot symbols on the basis of the original subblock $\underline{\mathbf{x}}$, each subblock will be expanded to include $N=n+P$ subcarriers, where $P$ is the number of pilot symbols, which is not smaller than the number of channel taps $L$. The final transmitted signal can be expressed as $\mathbf{x}=\left\{p_{1}, x_{1}, \ldots, p_{P}, \ldots, x_{n}\right\}$. Subsequent operations are same as the traditional OFDM systems.

Consider the MM-OFDM-IM systems operating over the multi-path Rayleigh fading channel. At the receiver, after the accurate time/frequency synchronization, the received signal in the frequency domain can be written as

$$
\mathbf{y}=\left[y_{1}, \ldots, y_{N}\right]^{T}=\mathbf{X F h}+\mathbf{w},
$$

where $\mathbf{X}=\operatorname{diag}(\mathbf{x})$ is the transmitted signal in the frequency domain, $\mathbf{h}=\left[h_{0}, \ldots, h_{L-1}\right]^{T}$ is CIR, and $\mathbf{F}$ is an $N \times L$ matrix, which is extracted from the first $L$ columns of the Fourier matrix of dimensions $N \times N$. $\mathbf{w}=\left[w_{1}, \ldots, w_{N}\right]^{T}$ is the additive white Gaussian noise (AWGN) vector over $N$ subcarriers, which has zero mean and covariance matrix $\mathcal{N}_{0} \mathbf{I}_{N}$.

Our goal is to recover the transmitted signal $\mathbf{x}$ directly from the observed received signal $y$ under the unobserved CIR $\mathbf{h}$ from (1). To this end, we can write the log-likelihood function associated with (1) as:

$$
\mathbf{L}(\mathbf{x})=\ln f(\mathbf{y}, \mathbf{h} \mid \mathbf{x}) .
$$

However, it is difficult to manipulate (2) directly due to the existence of the unobserved CIR $\mathbf{h}$. Therefore, we can use the EM algorithm, which is an iterative optimization strategy that can search for the unknown parameters in an iterative manner under the incomplete data or data sets with the unobserved data, to adapt to the MM-OFDM-IM signal model to solve this problem.

\subsection{Proposed EM detector for MM-OFDM-IM}

Motivated by the property of the EM algorithm that can effectively solve the problem lying in the detection when some useful information is well-hidden in the observed data, we aim to propose a novel detector by resorting to the EM algorithm for MM-OFDM-IM.

Here, in the MM-OFDM-IM systems, the incomplete data is $\mathbf{y}$ and the unobserved data is $\mathbf{h}$, such that the complete data can be expressed as $(\mathbf{y}, \mathbf{h})$. In each iteration, the EM algorithm contains two steps [21]:

E step:

$$
\begin{aligned}
Q\left(\mathbf{x} \mid \mathbf{x}^{(i)}\right) & =E_{\mathbf{h}}\left\{\mathbf{L}(\mathbf{x}) \mid \mathbf{y}, \mathbf{x}^{(i)}\right\} \\
& =E_{\mathbf{h}}\left\{\ln f(\mathbf{y}, \mathbf{h} \mid \mathbf{x}) \mid \mathbf{y}, \mathbf{x}^{(i)}\right\},
\end{aligned}
$$

where the superscript ${ }^{(i)}$ means the $i$ th iteration. M step:

$$
\mathbf{x}^{(i+1)}=\underset{\mathbf{x}}{\arg \max } Q\left(\mathbf{x} \mid \mathbf{x}^{(i)}\right) .
$$

The E step is to calculate the mathematical expectation of the log-likelihood function $\mathbf{L}(\mathbf{x})$ for the complete data conditioned on the initial value or the previous iteration estimate $\mathbf{x}^{(i)}$ and the observed data $\mathbf{y}$ by averaging over the unobserved data $\mathbf{h}$. The $M$ step is to obtain a new $\mathbf{x}^{(i+1)}$ by maximizing $Q\left(\mathbf{x} \mid \mathbf{x}^{(i)}\right)$. Repeat the above two steps until $\mathbf{x}^{(i+1)}=\mathbf{x}^{(i)}$, where we consider the EM algorithm has converged and $\mathbf{x}^{(i+1)}$ turns out to be the optimal value of the desired unknown parameter.

Next, we carry out a further analysis of the E step and $M$ step, respectively.

For MM-OFDM-IM systems, the information embedded into the transmitted signal contains the information symbols part and the mode permutations part, which implies that different combinations of these two parts will result in diverse transmitted signals. Obviously, if we intuitively solve the E step (3) by trying all possible realizations of the transmitted signal by jointly considering all possible mode permutations and constellation points, the computational complexity will be prohibitive. To circumvent this problem, we first confine the search space to a relatively small zone by assuming a specific mode permutation and then traverse the whole search space by relaxing the assumption.

Assume the $k$ th mode permutation is employed, where $k \in\{1, \ldots, K\}$ with $K=2^{m_{1}}$. In this case, the E step in (3) 
can be further turned into

$$
\begin{aligned}
Q\left(\mathbf{x}_{k} \mid \mathbf{x}_{k}^{(i)}\right) & =E_{\mathbf{h}}\left\{\ln \frac{f\left(\mathbf{y}, \mathbf{x}_{k}, \mathbf{h}\right)}{\operatorname{Pr}\left(\mathbf{x}_{k}\right)} \mid \mathbf{y}, \mathbf{x}_{k}^{(i)}\right\} \\
& =E_{\mathbf{h}}\left\{\ln \frac{f\left(\mathbf{y}, \mathbf{x}_{k}, \mathbf{h} \mid J_{k}\right) \operatorname{Pr}\left(J_{k}\right)}{\operatorname{Pr}\left(J_{k}\right) \operatorname{Pr}\left(\mathbf{x}_{k} \mid J_{k}\right)} \mid \mathbf{y}, \mathbf{x}_{k}^{(i)}\right\} \\
& =E_{\mathbf{h}}\left\{\ln \frac{f\left(\mathbf{y}, \mathbf{x}_{k}, \mathbf{h} \mid J_{k}\right)}{\operatorname{Pr}\left(\mathbf{x}_{k} \mid J_{k}\right)} \mid \mathbf{y}, \mathbf{x}_{k}^{(i)}\right\},
\end{aligned}
$$

In light of (4), the M step can be performed to obtain the transmitted signal estimate at the $(i+1)$ th iteration by maximizing (5) given $\mathbf{x}_{k}^{(i)}$,

$$
\begin{aligned}
\mathbf{x}_{k}^{(i+1)} & =\underset{\mathbf{x}_{k}}{\arg \max } Q\left(\mathbf{x}_{k} \mid \mathbf{x}_{k}^{(i)}\right) \\
& =\underset{\mathbf{x}_{k}}{\arg \max } E_{\mathbf{h}}\left\{\ln \frac{f\left(\mathbf{y}, \mathbf{x}_{k}, \mathbf{h} \mid J_{k}\right)}{\operatorname{Pr}\left(\mathbf{x}_{k} \mid J_{k}\right)} \mid \mathbf{y}, \mathbf{x}_{k}^{(i)}\right\} .
\end{aligned}
$$

Since $J_{k}$ and $\mathbf{x}_{k}$ are selected by the two parts of the incoming bits $m_{1}$ and $m_{2}$, and the signal constellation points are also drawn equiprobably, (6) can be further simplified as follows

$$
\mathbf{x}_{k}^{(i+1)}=\underset{\mathbf{x}_{k}}{\arg \max } E_{\mathbf{h}}\left\{\ln \left(f\left(\mathbf{y}, \mathbf{x}_{k}, \mathbf{h}\right)\right) \mid \mathbf{y}, \mathbf{x}_{k}^{(i)}\right\} .
$$

It can be readily figured out that (7) has almost the same form as the EM problem formulation for the transmitted signal estimate in the conventional OFDM systems. On this issue, the only difference between the conventional OFDM systems and MM-OFDM-IM systems is that the latter imposes an additional condition on the transmitted signal format, which requires the modulated symbols to be drawn from $n$ modes with a permutation dictated by $J_{k}$. For the conventional OFDM systems without any constraint on the transmitted signal format, an elegant solution is available in [[22], Eq. (36)]. Applying a similar idea to [22], the transmitted signal of MM-OFDM-IM systems under the $k$ th mode permutation can be estimated in a closed form as

$$
\tilde{\mathbf{x}}_{k}^{(i+1)}=\left(\mathbf{y}^{H} \mathbf{X}_{k}^{(i)} \mathbf{F} \mathbf{F}^{H} \mathbf{Y}\right)^{T},
$$

where $\mathbf{Y}=\operatorname{diag}(\mathbf{y})$ and $\mathbf{X}_{k}^{(i)}=\operatorname{diag}\left(\mathbf{x}_{k}^{(i)}\right)$, and (8) is also the final form of the $\mathrm{M}$ step.

On the other hand, the minimum mean square error (MMSE) estimation of the CIR [22] at the $(i+1)$ th iteration can be computed by

$$
\mathbf{h}_{k}^{(i+1)}=\Gamma^{(i+1)}\left(\frac{2}{N_{0}} \mathbf{F}^{H}\left(\tilde{\mathbf{X}}_{k}^{(i+1)}\right)^{H} \mathbf{y}+\Gamma^{-1} E(\mathbf{h})\right),
$$

where $\tilde{\mathbf{X}}_{k}^{(i+1)}=\operatorname{diag}\left(\tilde{\mathbf{x}}_{\mathbf{k}}^{(i+1)}\right)$, and

$$
\Gamma^{(i+1)}=\left(\frac{2}{N_{0}} \mathbf{F}^{H}\left(\tilde{\mathbf{X}}_{k}^{(i+1)}\right)^{H} \tilde{\mathbf{X}}_{k}^{(i+1)} \mathbf{F}+\Gamma^{-1}\right)^{-1}
$$

is the posterior covariance matrix of the CIR at the $(i+$ 1)th iteration.
At this point, with (8) and taking into account the constraint on the transmitted signal format, we can solve (7) as

$$
\mathbf{x}_{k}^{(i+1)}=\underset{\mathbf{x}_{k}}{\arg \min }\left\|\mathbf{x}_{k}-\tilde{\mathbf{x}}_{k}^{(i+1)}\right\|^{2} .
$$

Note that (11) is in essence a quantization operation, which quantizes $\tilde{\mathbf{x}}_{k}^{(i+1)}$ to the closest constellation points of the modes with a permutation $J_{k}$, and it can be realized subcarrier by subcarrier to save the computations.

Performing the above iterative operation between E step and $M$ step until the EM algorithm converges, the transmitted signal estimation under the $k$ th mode permutation can be eventually obtained, denoted by $\hat{\mathbf{x}}_{k}:=\mathbf{x}_{k}^{(i+1)}$. To account for its estimation accuracy, we introduce a likelihood metric by borrowing the Euclidean distance:

$$
\xi_{k}=\left\|\mathbf{y}-\hat{\mathbf{X}}_{k} \mathbf{F} \hat{\mathbf{h}}_{k}\right\|^{2},
$$

where $\hat{\mathbf{X}}_{k}=\operatorname{diag}\left\{\hat{\mathbf{x}}_{k}\right\}$ and $\hat{\mathbf{h}}_{k}$ is the final MMSE estimation of the CIR obtained by $\hat{\mathbf{h}}_{k}:=\mathbf{h}_{k}^{(i+1)}$. By definition, it is clear that a smaller $\xi_{k}$ means a higher probability that $\hat{\mathbf{x}}_{k}$ could be the real transmitted signal in (1).

Expanding the search space to perform the same procedure described above for all $K$ mode permutations, we can assemble all transmitted signal estimates under $K$ mode permutations into a candidate set $\left\{\hat{\mathbf{x}}_{1}, \ldots, \hat{\mathbf{x}}_{K}\right\}$. After that, the index of the most-likely mode permutation can be easily determined by

$$
\hat{k}=\underset{k}{\arg \min } \xi_{k} \text {. }
$$

Finally, the most-likely transmitted signal can be estimated as $\hat{\mathbf{x}}_{\hat{k}}$, which is corresponding to the $\hat{k}$ th mode permutation derived by (13).

For better understanding, we summarize the aforementioned entire procedure in Algorithm 1 with one subblock.

In addition, it is worth mentioning that the proposed EM detector can be also applied to the DM-OFDM-IM systems. Since the symbol generation schemes of DMOFDM-IM systems is slightly different from MM-OFDMIM systems, thus, some adjustments need to be made to the above EM detector. Consider DM-OFDM-IM systems with $n$ subcarriers, then divide the $n$ subcarriers into two index subsets, namely $A$ and $B$ with the sizes $g$ and $n-g$, respectively. These two index subsets will be modulated by two different constellation sets of $\mathcal{M}_{A}$ and $\mathcal{M}_{B}$. Different from MM-OFDM-IM systems, redefine $J=\left\{a_{1}, b_{1}, \ldots, a_{g}, b_{n-g}\right\}$ based on the symbol generation scheme of DM-OFDM-IM, where $\left\{a_{1}, \ldots, a_{g}\right\} \in A$ and $\left\{b_{1}, \ldots, b_{n-g}\right\} \in B$. In this context, the remaining operations of the proposed EM detector are same as the MM-OFDM-IM systems. 


\subsection{Algorithm initialization}

As is well known, the initial value is critical for the EM algorithm since the EM algorithm is sensitive to the initial value. If it is not properly chosen, the algorithm may probably output a solution that is not globally optimal. Therefore, for each subblock, it is recommended to perform the least squares (LS) channel estimation [23], which can be implemented with aid of the pilot symbols inserted ahead of or between the data symbols. Assuming that the pilot symbols positions in each subblock are included in a set $\mathbb{Z}=\left\{z_{1}, z_{2}, \ldots, z_{P}\right\}$, the channel coefficients corresponding to the pilot symbols in the frequency domain can be written as

$$
h_{p}^{f}=\frac{\mathbf{y}\left(z_{p}\right)}{\mathbf{p}\left(z_{p}\right)}, p=1, \ldots, P .
$$

Then, the channel coefficients corresponding to the data symbols in the frequency domain can be obtained from those associated with the pilot symbols via discrete Fourier transform interpolation. Specifically, we first perform inverse fast Fourier transform (IFFT) on $\mathbf{h}_{P}^{f}=$ $\left\{h_{1}^{f}, h_{2}^{f}, \ldots, h_{P}^{f}\right\}$ to obtain the initial time domain CIR by

$$
\mathbf{h}^{t}=\mathbf{F}_{P}^{H} \mathbf{h}_{P}^{f},
$$

where $\mathbf{F}_{P}$ is the Fourier matrix of dimensions $P \times P$. Next, perform FFT on (15) for all $N$ subcarriers to get the channel coefficients in frequency domain, which can be expressed as

$$
\mathbf{h}_{N}^{f}=\mathbf{F}_{N \times P} \mathbf{h}^{t}
$$

where $\mathbf{F}_{N \times P}$ is the Fourier submatrix of dimensions $N \times P$, which is extracted from the Fourier matrix of $N \times N$ by taking its rows of indices equal to the positions of the pilot symbols.

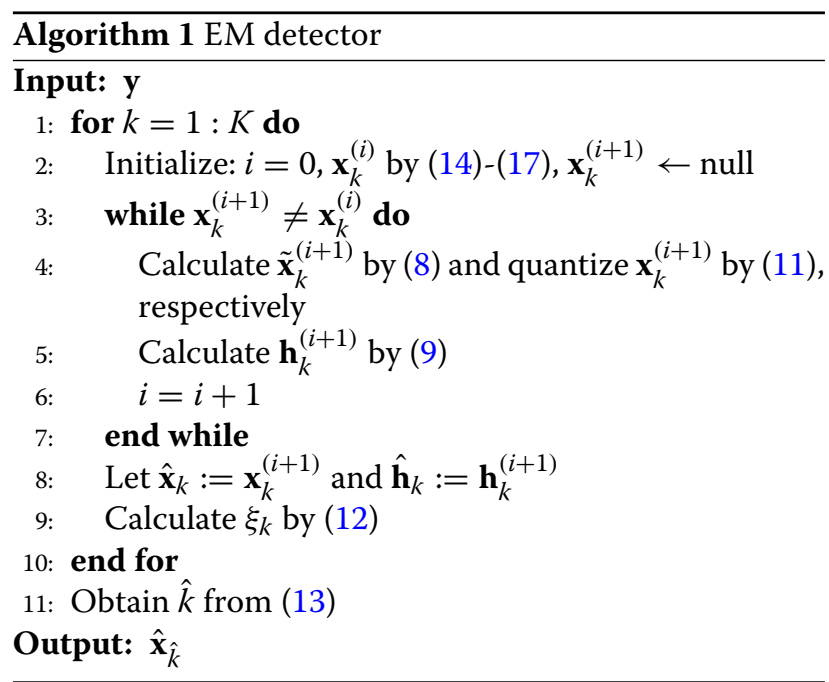

Using the estimated channel coefficients corresponding to positions of the data symbols, namely $\mathbb{Z}^{\prime}=$ $\left\{z_{1}^{\prime}, z_{2}^{\prime}, \ldots, z_{n}^{\prime}\right\}$, from (16), the initial transmitted signal estimate can be obtained by

$$
x_{d}^{(0)}=\frac{\mathbf{y}\left(z_{d}^{\prime}\right)}{\mathbf{h}_{N d}^{f}}, d=1, \ldots, n .
$$

Finally, $\underline{\mathbf{x}}^{(0)}=\left\{x_{1}^{(0)}, x_{2}^{(0)}, \ldots, x_{n}^{(0)}\right\}$ will be quantized to the $k$ th mode permutation $\mathbf{x}_{k}^{(0)}=\left\{p_{1}, x_{1}^{(0)}, \ldots, p_{P}, \ldots, x_{n}^{(0)}\right\}$ and served as the input of the EM algorithm for subsequent calculations.

\subsection{Complexity analysis}

As can be seen from the description of the proposed EM detector, for each subblock, the majority of the computation in the EM detector lies in (11), which is iteratively computed with a number of calculations depending on the iteration times. Since there are $n$ subcarriers in each subblock, (11) involves $n$ parallel computations, each of which searches over $M$ constellation points. In addition, each subblock needs to traverse in total $n$ ! mode permutations. Therefore, the computational complexity of the proposed EM detector is of order $\sim \mathcal{O}(\bar{i} n M n !)$ per subblock, where $\bar{i}$ is the average number of iterations.

Table 1 lists the computational complexity comparision per subblock for the EM, ML, LCML, and SW detectors [12]. Compared with the SW and LCML detectors, the ML and EM detectors both have higher computational complexity. For the ML and the proposed EM detectors, it is difficult to tell exactly the winner between them in terms of computational complexity since $\bar{i}$ is a random variable. However, it is predicted that for a larger value of $M$ or $n$, the proposed EM detector is more likely to have lower computational complexity than the ML detector. In addition, we will measure the computational complexity by the average number of iterations $\bar{i}$ in the simulation section to further determine its impact on the computational complexity of the EM detector.

\section{Results and discussion}

In this section, we conduct Monte Carlo computer simulations to verify the effectiveness of the proposed EM detector and measure the average number of iterations

Table 1 Computational complexity comparision per subblock for $E M, M L, L C M L$, and SW detectors

\begin{tabular}{ll}
\hline Detector & Complexity \\
\hline EM & $\mathcal{O}($ in Mn! $)$ \\
ML & $\mathcal{O}\left(n ! M^{n}\right)$ \\
LCML & $\mathcal{O}\left(M n^{2}+n !\right)$ \\
SW & $\mathcal{O}\left(M n^{2} / 2+M n / 2\right)$ \\
\hline
\end{tabular}


required for the convergence of the EM detector versus the power ratio of a bit and the noise, namely $E_{b} / N_{0}$. The performance of the ML, EM, SW, and LCML detectors for MM-OFDM-IM from the perspective of BER are presented under the Rayleigh fading channel with $L=4$ channel taps. Assume that the channel is quasistatic, which implies the CIR is constant within an OFDM symbol transmission period and varies for the next transmission period. In the BER simulations, there are in total 100,000 subblocks and $n=8$ subcarriers carrying data symbols in each subblock. In addition, $P=4$ pilot symbols are equally inserted into the data symbols. The initial value of the transmitted signal obtained during the algorithm initialization are used for the ML, SW, and LCML detections and the initialization in the EM detection. For the simulation of the number of iterations, we average the number of iterations under all possible mode permutations with $n=8$ subcarriers carrying data symbols in each subblock for BPSK and QPSK modulation.

Figure 2 shows the comparison results of ML, EM, SW, and LCML detectors in terms of BER with the BPSK and QPSK modulation for MM-OFDM-IM. From Fig. 2 we can clearly see that the BER performance of the EM detector is better than the other three detectors. Compared to LCML detector in [12], the ML and EM detectors both obtain significant SNR gains at an SNR region between 18 and $33 \mathrm{~dB}$ for BPSK and QPSK modulation for the reason that the LCML detector reduces the search space and the total number of metric calculations in the detection process. Compared to SW detector in [12], the ML and EM

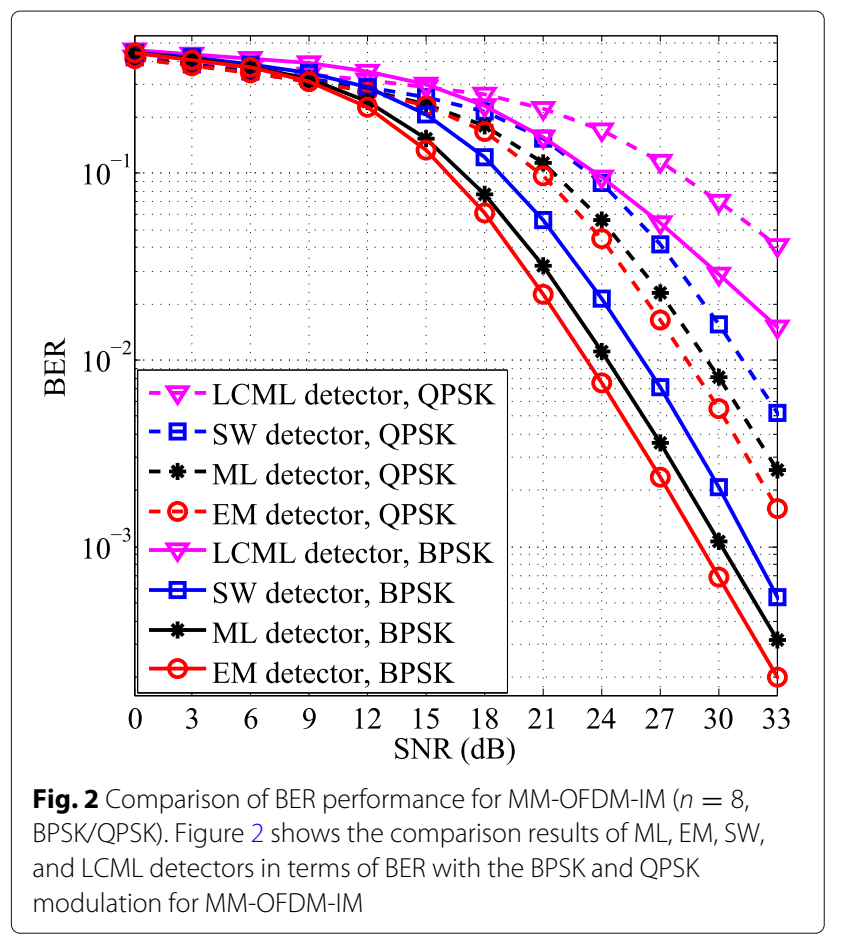

detectors obtain up to $1.6 \mathrm{~dB}$ and $2.8 \mathrm{~dB}$ SNR gains at an SNR region between 18 and $33 \mathrm{~dB}$ for BPSK modulation, respectively. The efficiency of the SW detector arises from the error propagation.

Figure 2 also indicates that the proposed EM detector outperforms the ML detector, obtaining an up to $1.2 \mathrm{~dB}$ SNR gains at an SNR region between 18 and $33 \mathrm{~dB}$. This can be understood in the following. Recall that both the ML and EM detectors use the same initial value of the transmitted signal obtained by the LS channel estimation based on the pilot symbols at the algorithm initialization step. However, the ML detector only performs ML detection based on the estimated CIR and the initial value of the transmitted signal obtained during the algorithm initialization step, while in the proposed EM detector, the estimated CIR and the initial value of the transmitted signal are not only obtained from the algorithm initialization step but also iteratively updated in subsequent calculations. From the previous description of the proposed EM detector, we can see that (8) is iteratively estimated for the transmitted signals, which means a more accurate transmitted signal estimate will be obtained, and the CIR based on the current transmitted signal estimate in (8) can also be updated by (9) to further improve the accuracy of CIR estimation. Therefore, there is no doubt that the likelihood metric in (12), which is a joint decision of the transmitted signal and CIR, will be more reliable. Consequently, the final BER performance obtained by the proposed EM detector is better than that of the ML detector.

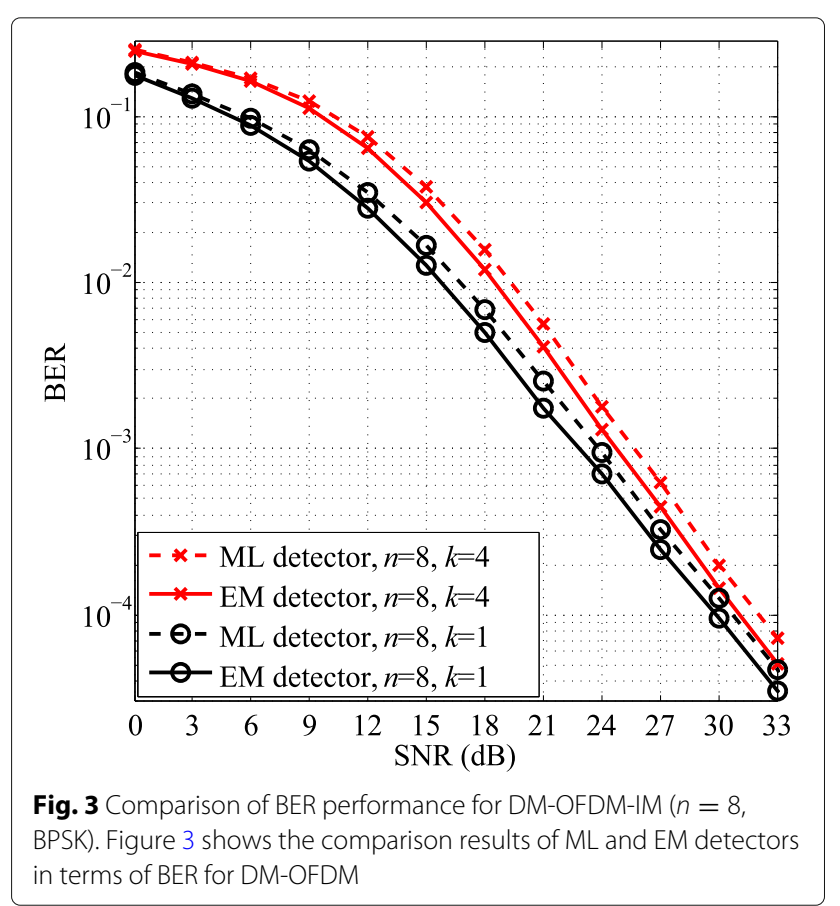


Figure 3 shows the comparison results of ML and EM detectors in terms of BER for DM-OFDM, where $k=1$ or $k=4$ subcarriers are modulated by rotated BPSK. As can be seen in Fig. 3, the proposed EM detector also obtains an up to $1.2 \mathrm{~dB}$ SNR gains, which indicates that the proposed EM detector can be applied to DM-OFDM-IM systems.

Figure 4 shows the average number of iterations versus $E_{b} / N_{0}$ of EM detector with BPSK and QPSK modulation for MM-OFDM-IM. As can be seen from Fig. 4, the average number of iterations of the EM detector is small in a wide range of SNR regions and decreases with increasing SNR. Even at low SNR, the average number of iterations is less than 2, which indicates the computational complexity of the EM detector is less affected by the average number of iterations. At this point, we can finally conclude that the computational complexity of the proposed EM detector is lower than ML detector.

\section{Conclusion}

In this paper, a novel EM detector for MM-OFDM-IM systems has been proposed. The proposed EM detector uses the EM algorithm to build a candidate set of the transmitted signal constellation points for each subblock by trying all possible mode permutations. Subsequently, the minimum Euclidean distance criterion is used to determine the most-likely signal constellation points and the corresponding mode permutations from the candidate set. Simulation results have verified that the proposed EM detector only needs a small number of iterations to converge and it outperforms the ML, SW, and LCML detectors in terms of BER.

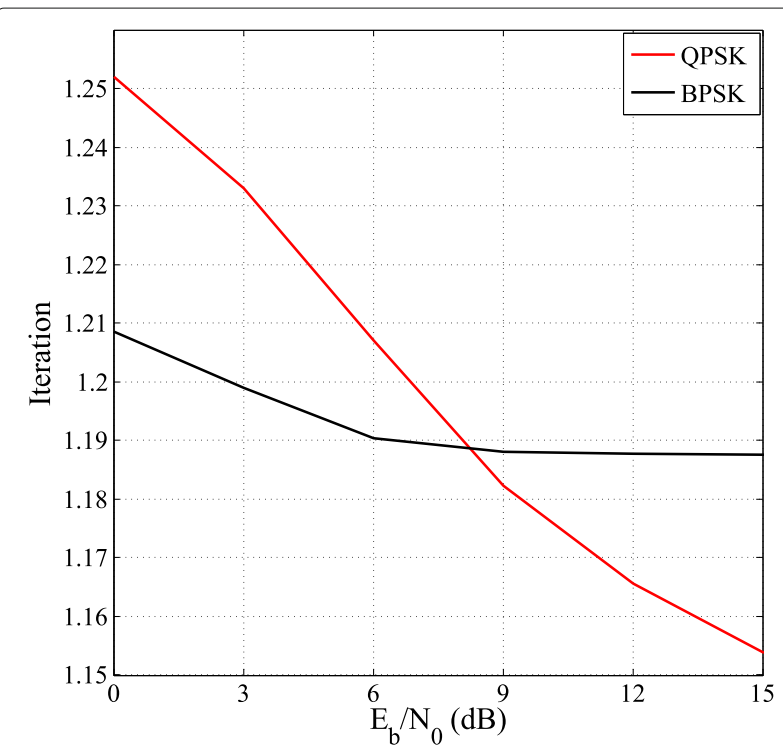

Fig. 4 The average number of iterations versus $E_{b} / N_{0}$ of EM detector for MM-OFDM-IM ( $n=8$, BPSK/QPSK). Figure 4 shows the average number of iterations versus $E_{b} / N_{0}$ of EM detector with BPSK and QPSK modulation for MM-OFDM-IM

\section{Abbreviations}

AWGN: Additive white Gaussian noise; BER: Bit error rate; ML:

Maximum-likelihood; CIR: Channel impulse response; OFDM: Orthogonal frequency division multiplexing; IM: Index modulation; OFDM-IM: OFDM with IM; DM-OFDM-IM: Dual-mode OFDM-IM; EM: Expectation maximization; FFT: Fast Fourier transform; IFFT: Inverse fast Fourier transform; LLR: Log-likelihood ratio; LCML: Low-complexity maximum-likelihood; LS: Least-squares; MM-OFDM-IM: Multiple-mode OFDM with IM; MIMO-OFDM-IM: Multiple-input multiple-output OFDM with IM; MMSE: Minimum mean square error; IQ: In-phase and quadrature; PSK: Phase shift keying; SNR: Signal-to-noise ratio; SW: Subcarrier-wise

\section{Acknowledgements}

Not applicable.

\section{Authors' contributions}

$\mathrm{HQ}$ is in charge of the simulations and wrote the manuscript. MW designed the initial idea. HY, FC, and FJ helped to improve the writing of this paper. The author(s) read and approved the final manuscript.

\section{Funding}

This work was supported in part by the National Natural Science Foundation of China under Grants U1809211, 61771202, 61671211, and 61871190

Availability of data and materials

Not applicable.

\section{Competing interests}

The authors declare that they have no competing interests.

Received: 27 September 2019 Accepted: 20 February 2020

Published online: 10 March 2020

\section{References}

1. M. Wen, X. Cheng, L. Yang, Index modulation for $5 G$ wireless communications. (Springer, Berlin, 2017)

2. E. Basar, M. Wen, R. Mesleh, M. Di Renzo, Y. Xiao, H. Haas, Index modulation techniques for next-generation wireless networks. IEEE Access. 5, 16693-16746 (2017)

3. R. Y. Mesleh, H. Haas, S. Sinanovic, C. W. Ahn, S. Yun, Spatial modulation. IEEE Trans. Veh. Technol. 57(4), 2228-2241 (2008)

4. E. Basar, Index modulation techniques for $5 \mathrm{~g}$ wireless networks. IEEE Commun. Mag. 54(7), 168-175 (2016)

5. E. Basar, U. Aygolu, E. Panayirci, H. V. Poor, Orthogonal frequency division multiplexing with index modulation. IEEE Trans. Signal Process. 61(22), 5536-5549 (2013)

6. M. Wen, X. Cheng, M. Ma, B. Jiao, H. V. Poor, On the achievable rate of OFDM with index modulation. IEEE Trans. Signal Process. 64(8), 1919-1932 (2016)

7. M. Wen, B. Ye, E. Basar, Q. Li, F. Ji, Enhanced orthogonal frequency division multiplexing with index modulation. IEEE Trans. Wirel. Commun. 16(7), 4786-4801 (2017)

8. M. Wen, Y. Zhang, J. Li, E. Basar, F. Chen, Equiprobable subcarrier activation method for OFDM with index modulation. IEEE Commun. Lett. 20(12), 2386-2389 (2016)

9. R. Fan, Y. J. Yu, Y. L. Guan, Generalization of orthogonal frequency division multiplexing with index modulation. IEEE Trans. Wirel. Commun. 14(10), 5350-5359 (2015)

10. E. Basar, On multiple-input multiple-output OFDM with index modulation for next generation wireless networks. IEEE Trans. Signal Process. 64(15), 3868-3878 (2016)

11. T. Mao, Z. Wang, Q. Wang, S. Chen, L. Hanzo, Dual-mode index modulation aided OFDM. IEEE Access. 5, 50-60 (2017)

12. M. Wen, E. Basar, Q. Li, B. Zheng, M. Zhang, Multiple-mode orthogonal frequency division multiplexing with index modulation. IEEE Trans. Commun. 65(9), 3892-3906 (2017)

13. B. Zheng, F. Chen, M. Wen, F. Ji, H. Yu, Y. Liu, Low-complexity ML detector and performance analysis for OFDM with in-phase/quadrature index modulation. IEEE Commun. Lett. 19(11), 1893-1896 (2015)

14. B. Zheng, M. Wen, E. Basar, F. Chen, Multiple-input multiple-output OFDM with index modulation: low-complexity detector design. IEEE Trans. Signal Process. 65(11), 2758-2772 (2017) 
15. A. Goldsmith, Wireless Communications. (Cambridge university press, England, 2005)

16. P. Chen, H. Kobayashi, in 2002 IEEE International Conference on Communications. Conference Proceedings. ICC 2002 (Cat. No.02CH37333), vol. 3. Maximum likelihood channel estimation and signal detection for OFDM systems, (2002), pp. 1640-16453. https://doi.org/10.1109/icc.2002. 997127

17. A. P. Dempster, N. M. Laird, D. B. Rubin, Maximum likelihood from incomplete data via the EM algorithm. J. R. Stat. Soc. Ser. B (Methodol.) 39 $1-38$ (1977)

18. E. Ustunel, in 2012 IEEE Symposium on Computers and Communications (ISCC). Expectation-maximization (EM) based channel estimation and interpolation in OFDM systems, (2012), pp. 166-170. https://doi.org/10. 1109/iscc.2012.6249287

19. S. Park, J. W. Choi, J. Seol, B. Shim, Expectation-maximization-based channel estimation for multiuser MIMO systems. IEEE Trans. Commun. 65(6), 2397-2410 (2017)

20. X. Wang, G. Wang, R. Fan, B. Ai, Channel estimation with expectation maximization and historical information based basis expansion model for wireless communication systems on high speed railways. IEEE Access. $\mathbf{6}$ 72-80 (2018)

21. T. K. Moon, The expectation-maximization algorithm. IEEE Signal Process. Mag. 13(6), 47-60 (1996)

22. X. Ma, H. Kobayashi, S. C. Schwartz, EM-based channel estimation algorithms for OFDM. EURASIP J. Adv. Signal Process. 2004, 1460-1477 (2004)

23. M. K. Ozdemir, H. Arslan, Channel estimation for wireless OFDM systems. IEEE Commun. Surv. Tutor. 9(2), 18-48 (2007)

\section{Publisher's Note}

Springer Nature remains neutral with regard to jurisdictional claims in published maps and institutional affiliations.

\section{Submit your manuscript to a SpringerOpen ${ }^{\circ}$ journal and benefit from:}

- Convenient online submission

Rigorous peer review

- Open access: articles freely available online

- High visibility within the field

- Retaining the copyright to your article

Submit your next manuscript at $\gg$ springeropen.com 